PROCEEDINGS OF THE

AMERICAN MATHEMATICAL SOCIETY

Volume 133, Number 7 , Pages $2147-2155$

S 0002-9939(05)07751-8

Article electronically published on January 31, 2005

\title{
THE LINEAR SPACE OF GENERALIZED BROWNIAN MOTIONS WITH APPLICATIONS
}

\author{
JEONG HYUN LEE
}

(Communicated by Richard C. Bradley)

\begin{abstract}
In this paper, we define, motivated by recent works of Chang and Skoug, stochastic integrals for a generalized Brownian motion $(\mathrm{gBm}) X$ and then use it to study the representation problem on the linear space $H(X)$ spanned by $X$. We next establish a translation theorem for $L^{p}$-functionals of $X, p \geq 1$, and then use this translation to establish an integration by parts formula for $L^{p}$-functionals of $X$.
\end{abstract}

\section{INTRODUCTION}

Let $\left(C_{0}[0, T], \mathcal{B}\left(C_{0}[0, T]\right), m_{w}\right)$ be the Wiener measure space. Let $a \in C_{0}[0, T]$ be of bounded variation on $[0, T]$ and let $b \in C_{0}[0, T]$ be strictly increasing and of bounded variation on $[0, T]$. Then by Theorem $14.2([12$, p. 187) there exists a Gaussian measure $\mu$ on $\left(C_{0}[0, T], \mathcal{B}\left(C_{0}[0, T]\right)\right)$ such that the coordinate process defined as $X(t, x)=x(t)$ is a continuous additive process on $\left(C_{0}[0, T], \mathcal{B}\left(C_{0}[0, T]\right), \mu\right)$ and $[0, T]$ on which the probability distribution of $X\left(t_{2}, \cdot\right)-X\left(t_{1}, \cdot\right), t_{1}<t_{2}$ is normally distributed with mean $a\left(t_{2}\right)-a\left(t_{1}\right)$ and variance $b\left(t_{2}\right)-b\left(t_{1}\right)$.

Such a process $X=\left(X_{t}, t \in[0, T]\right)$ will be referred to as the generalized Brownian motion $(\mathrm{gBm})$ determined by the mean function $a(t)$ and the variance function $b(t)$. We will write the space $\left(C_{0}[0, T], \mathcal{B}\left(C_{0}[0, T]\right), \mu\right)$ as $\left(C_{a, b}[0, T], \mathcal{B}\left(C_{a, b}[0, T]\right), \mu\right)$. We note that the Wiener process $W(t, x)=x(t)$ on $\left(C_{0}[0, T], \mathcal{B}\left(C_{0}[0, T]\right), m_{w}\right)$ and $[0, T]$ is free of drift and is stationary in time, while the process $X=\left(X_{t}, t \in[0, T]\right)$ on $\left(C_{a, b}[0, T], \mathcal{B}\left(C_{a, b}[0, T]\right), \mu\right)$ is subject to the drift $a(t)$ and is nonstationary in time, and can be used to describe the Black-Scholes model with time-dependent drift and diffusion coefficient in the financial market, as well as the model of interest rates and bond prices. For more details about the Black-Scholes model with timedependent parameters, see e.g. (9], p. 80, p. 87). For the model of bond prices and interest rates, see e.g. (9], p. 125, p. 127).

Let $X=\left(X_{t}, t \in[0, T]\right)$ be a $\mathrm{gBm}$ determined by $a(t)$ and $b(t)$. Then we note that $X$ is an $L^{2}$-process and $\sigma(X)=\mathcal{B}\left(C_{a, b}[0, T]\right)$. There are two Hilbert spaces associated to the $\mathrm{gBm} X$. The one is the nonlinear space of $X, L^{2}(X)=$ $L^{2}\left(C_{a, b}[0, T], \sigma(X), \mu\right)$ (consisting of all $\sigma(X)$-measurable random variables with

Received by the editors January 20, 2004 and, in revised form, March 19, 2004.

2000 Mathematics Subject Classification. Primary 60J65, 28C20.

Key words and phrases. Generalized Brownian motion, translation theorem, directional derivative, integration by parts formula.

This work was supported by Korea Research Foundation Grant (KRF-2003-015-C00065).

(C)2005 American Mathematical Society 
finite second moment). Elements of $L^{2}(X)$ are called (nonlinear) $L^{2}$-functionals of $X$. The second Hilbert space is the linear space $H(X)$ of $X$, which is the closed subspace of $L^{2}(X)$ spanned by $X$. Elements of $H(X)$ are called linear $L^{2}$ functionals of $X$.

Let $\tilde{X}=\left(\tilde{X}_{t} \equiv X_{t}-a(t), t \in[0, T]\right)$. Let $L^{2}(\tilde{X})(H(\tilde{X})$, resp. $)$ denote the nonlinear (linear, resp.) space of $\tilde{X}$.

The authors in [4, 5] used a gBm to define a generalized analytic Feynman integral and a generalized analytic Fourier-Feynman transform and then studied the first variations and the integration by parts formula involving these integrals and transforms. Motivated by these recent works (4], 5]), in this paper we introduce a stochastic integral for the $\mathrm{gBm} X$ for which the space of the integrands is wider than the one for the stochastic integrals defined in [4], and then use it to study the representation problem on the linear space $H(X)$. We next establish a translation theorem for $L^{p}$-functionals, $p \geq 1$, which is proved by using a different method from the one given in [3], [5] and then use this translation theorem to establish an integration by parts formula for $L^{p}$-functionals of $X$ with $p \geq 1$.

The organization of this paper is as follows. In Section 2, we introduce the function Hilbert space $L_{a, b}^{1,2}[0, T]$, which is our choice of function Hilbert spaces to represent the linear space $H(X)$. In Section 3, we establish a translation theorem for $L^{p}$-functionals, $p \geq 1$. In Section 4 , we use the translation theorem obtained in Section 3 to establish an integration by parts formula for the functionals in $L^{p}(X)$, $p \geq 1$.

\section{Stochastic integration For GBM}

In this section we introduce the function Hilbert space $L_{a, b}^{1,2}[0, T]$, which will be our choice of function Hilbert spaces for the linear representation problem on the $\operatorname{gBm} X$.

We will assume throughout this paper that $a(t)$ is an absolutely continuous function on $[0, T]$ with $a(0)=0$ and $a^{\prime}(t) \in L^{2}[0, T]$, and $b(t)$ is a differentiable function with $b(0)=0$ and $b_{1} \leq b^{\prime}(t) \leq b_{2}\left(b_{1}, b_{2}>0\right)$ for all $t \in[0, T]$. This assumption is slightly milder than the one given in [4].

Let $L_{b}^{2}[0, T]$ be the Hilbert space of functions on $[0, T]$ given by

$$
L_{b}^{2}[0, T]=\left\{f \in L^{2}[0, T] \mid \int_{0}^{T} f^{2}(s) d b(s)<\infty\right\}
$$

equipped with an inner product

$$
\langle f, g\rangle_{b}=\int_{0}^{T} f(s) g(s) d b(s) .
$$

Let $L_{a, b}^{1,2}[0, T]$ be the Hilbert space of functions on $[0, T]$ defined by

$$
L_{a, b}^{1,2}[0, T]=\left\{f \in L_{b}^{2}[0, T]\left|\int_{0}^{T}\right| f(s)|d| a \mid(s)<\infty\right\}
$$

equipped with an inner product

$$
\langle f, g\rangle_{a, b}=\int_{0}^{T} f(s) g(s) d b(s)+\int_{0}^{T} f(s) d a(s) \int_{0}^{T} g(s) d a(s)
$$


where $|a|(t)=\int_{0}^{t}|d a|, t \in[0, T]$. It is easy to see that $\|f\|_{a, b}=0$ if and only if $f=0$ a.e. on $[0, T]$ and that the three norms $\|\cdot\|,\|\cdot\|_{b}$ and $\|\cdot\|_{a, b}$ are equivalent. Hence $L^{2}[0, T], L_{b}^{2}[0, T]$ and $L_{a, b}^{1,2}[0, T]$ coincide as sets, and furthermore, $L_{a, b}^{1,2}[0, T]$ is a separable Hilbert space.

Let $\mathcal{S}$ be the set of all step functions on $[0, T], f=\sum_{j=0}^{n-1} c_{j} 1_{\left(t_{j}, t_{j+1}\right]}$, where $0=t_{0}<t_{1}<t_{2}<\cdots<t_{n}=T$ and $c_{j} \in \mathbb{R}$. We define $\theta: \mathcal{S} \rightarrow H(X)$ by

$$
\theta(f)=\sum_{j=0}^{n-1} c_{j}\left(X_{t_{j+1}}-X_{t_{j}}\right)=\sum_{j=0}^{n-1} c_{j}\left[\left(\tilde{X}_{t_{j+1}}-\tilde{X}_{t_{j}}\right)+\left(a\left(t_{j+1}\right)-a\left(t_{j}\right)\right)\right] .
$$

Clearly $\mathcal{S}$ is a linear dense subspace of $L_{a, b}^{1,2}[0, T]$ and for all $f, g \in \mathcal{S}$, we have the following identity:

$$
E[\theta(f)]=\int_{0}^{T} f d a \text { and } E[\theta(f) \theta(g)]=\langle f, g\rangle_{a, b},
$$

where $\int_{0}^{T} f d a$ denotes the Stieltjes integral of $f$ with respect to the absolutely continuous function $a$. Thus the correspondence $f \mapsto \theta(f)$ is an inner product preserving mapping from $\mathcal{S}$ to $H(X)$. Hence $\theta$ is uniquely extended to $L_{a, b}^{1,2}[0, T]$. For $f \in L_{a, b}^{1,2}[0, T]$, we call $\theta(f)$ the stochastic integral of $f$ against $X$ and write it as

$$
\theta(f)=\int_{0}^{T} f d X \text { or } \int_{0}^{T} f d \tilde{X}+\int_{0}^{T} f d a .
$$

Definition 2.1. The stochastic integral $\int_{0}^{T} f d X_{t}$ for $f \in L_{a, b}^{1,2}[0, T]$ is defined by

$$
\int_{0}^{T} f d X_{t}=\int_{0}^{T} f d \tilde{X}_{t}+\int_{0}^{T} f d a
$$

Proposition 2.2. Let $X$ be a $g B m$ determined by $a(t)$ and $b(t)$. Then

$$
L_{a, b}^{1,2}[0, T] \cong H(X)
$$

Proof. Let $\theta: \mathcal{S} \rightarrow H(X)$ be the mapping defined as above. Then $\theta\left(1_{(0, t]}\right)=$ $\int_{0}^{T} 1_{(0, t]}(u) d X(u)=X_{t}$ for any $t \in[0, T]$. But the set $\left\{X_{t} \mid t \in[0, T]\right\}$ generates $H(X)$. Hence the mapping $\theta$ is uniquely extended to a unitary isomorphism from $L_{a, b}^{1,2}[0, T]$ onto $H(X)$. This completes the proof.

Theorem 2.3. Let $X$ be a $g B m$ determined by $a(t)$ and $b(t)$. For $f, g \in L_{a, b}^{1,2}[0, T]$ and $\alpha, \beta \in \mathbb{R}$, the stochastic integral $\theta$ satisfies the following:

(1) $\theta(\alpha f+\beta g)=\alpha \theta(f)+\beta \theta(g)$.

(2) $E[\theta(f)]=\int_{0}^{T} f d a$.

(3) $\langle\theta(f), \theta(g)\rangle_{L^{2}(X)}=\langle f, g\rangle_{a, b}$.

(4) $\|\theta(f)\|_{L^{2}(X)}^{2}=\|f\|_{a, b}^{2}$.

(5) $\theta(f)$ is normally distributed with $N\left(\int_{0}^{T} f d a,\|f\|_{b}^{2}\right)$.

(6) $\left\{\theta(f), f \in L_{a, b}^{1,2}[0, T]\right\}$ is a Gaussian system of random variables. 
Proof. The proof of (1)-(4) has been done in Proposition 2.2

(5) For any $f \in L_{a, b}^{1,2}[0, T]$, there exists a sequence $f_{n} \in \mathcal{S}, n \geq 1$ such that $f_{n} \rightarrow f$. Then $\theta\left(f_{n}\right) \rightarrow \theta(f)$ in $L^{2}(X)$ and clearly the $\theta\left(f_{n}\right)$ 's are Gaussian variables. Hence the $L^{2}$-limit $\theta(f)$ of a sequence $\theta\left(f_{n}\right)$ is normally distributed with $N\left(\int_{0}^{T} f d a,\|f\|_{b}^{2}\right)$.

(6) The proof follows from the fact that for any finite linear combination of $\theta(f)$ 's, $\sum_{i=1}^{n} \alpha_{i} \theta\left(f_{i}\right)$ is normally distributed with $N\left(\sum_{i=1}^{n} \alpha_{i} \int_{0}^{T} f_{i} d a, \sum_{i, j=1}^{n} \alpha_{i} \alpha_{j}\left\langle f_{i}, f_{j}\right\rangle_{b}\right)$.

\section{TRANSLATION THEOREM FOR $L^{p}$-FUNCTIONALS}

Let $D$ be any set and $C$ be a real-valued function on $D \times D$. Then $C$ is called a covariance function on $D \times D$ if

(1) $C(s, t)=C(t, s)$,

(2) $\sum_{t, s \in I} a_{t} a_{s} C(t, s) \geq 0$ for all finite subsets $I$ of $D$ and $\left\{a_{s}, s \in I\right\} \subset \mathbb{R}$.

Let $C$ be a real-valued covariance function on $D \times D$. Then according to the following theorem due to Aronszajn [1, there exists a unique Hilbert space $K(C)$ of functions on $D$ satisfying the conditions in the following theorem.

Theorem 3.1. Let $D$ be any separable metric space and $C$ be a real-valued covariance function on $D \times D$. Then there exists a unique Hilbert space $K(C)$ of real-valued functions on $D$, with the inner product denoted by $\langle\cdot, \cdot\rangle_{K(C)}$ such that

(1) $C(t, \cdot) \in K(C)$, for each $t \in D$,

(2) $\langle f(\cdot), C(t, \cdot)\rangle_{K(C)}=f(t)$, for each $t \in D$ and $f \in K(C)$.

The space $K(C)$ in Theorem 3.1, is called the reproducing kernel Hilbert space (RKHS) of $C$. Indeed, $K(C)$ is defined as the closure of the linear span of the functions $\{C(t, \cdot) \mid t \in D\}$ with respect to the inner product $\langle C(t, \cdot), C(s, \cdot)\rangle_{K(C)}=$ $C(t, s)$. It is well known that if $C$ is continuous on $D \times D$, then $K(C)$ is a separable Hilbert space.

Proposition 3.2. Let $D=[0, T]$ and $C(t, s)=\min \{b(t), b(s)\}, s, t \in[0, T]$. Let $C_{b}^{\prime}[0, T]$ be the Hilbert space given by

$$
C_{b}^{\prime}[0, T]=\left\{\gamma \mid \gamma(t)=\int_{0}^{t} \frac{d \gamma}{d b} d b, \frac{d \gamma}{d b} \in L_{b}^{2}[0, T]\right\}
$$

equipped with the inner product defined by

$$
\left\langle\gamma_{1}, \gamma_{2}\right\rangle_{C_{b}^{\prime}[0, T]}=\left\langle\frac{d \gamma_{1}}{d b}, \frac{d \gamma_{2}}{d b}\right\rangle_{b},
$$

where $\frac{d \gamma}{d b} \equiv \frac{d \nu}{d \mu}$ with $\mu=$ the Borel-Stieltjes measure induced by $b$ and $\nu=$ the signed measure induced by $\gamma(t)=\int_{0}^{t} g d b, g \in L_{b}^{2}[0, T]$. Then $K(C)=C_{b}^{\prime}[0, T]$.

Proof. We first note that for all $s, t \in[0, T], C(t, s)=\min \{b(t), b(s)\}=\int_{0}^{T} 1_{[0, t \wedge s]} d b$ and $\frac{d C(t, \cdot)}{d b}=1_{[0, t]}(\cdot)$. Since $1_{[0, t]} \in L_{b}^{2}[0, T]$ for all $t \in[0, T]$, we have

$$
C(t, \cdot) \in C_{b}^{\prime}[0, T], \text { for each } t \in[0, T] .
$$


For any $f \in C_{b}^{\prime}[0, T]$ and for each $t \in[0, T]$, we have

$$
\begin{aligned}
\langle C(t, \cdot), f\rangle_{C_{b}^{\prime}[0, T]} & =\left\langle 1_{[0, t]}, \frac{d f}{d b}\right\rangle_{b} \\
& =\int_{0}^{t} \frac{d f}{d b} d b=f(t) .
\end{aligned}
$$

Hence by Theorem 3.1, $K(C)=C_{b}^{\prime}[0, T]$.

Proposition 3.3. Let $D=[0, T]$ and $C(t, s)=\min \{b(t), b(s)\}, s, t \in[0, T]$. Then we have $K(C) \cong L_{b}^{2}[0, T]$.

Proof. We define an operator $U$ from $L_{b}^{2}[0, T]$ to $C_{b}^{\prime}[0, T]$ by

$$
U f(t)=\int_{0}^{t} f(s) d b(s), f \in L_{b}^{2}[0, T] .
$$

Then we see that $\langle f, g\rangle_{b}=\left\langle\int_{0}^{t} f(s) d b(s), \int_{0}^{t} g(s) d b(s)\right\rangle_{C_{b}^{\prime}[0, T]}$. Thus $U$ is a unitary operator from $L_{b}^{2}[0, T]$ onto $C_{b}^{\prime}[0, T]$. Hence $K(C)=C_{b}^{\prime}[0, T] \cong L_{b}^{2}[0, T]$.

For $p \geq 1$, let $L^{p}(X) \equiv L^{p}\left(C_{a, b}[0, T], \mathcal{B}\left(C_{a, b}[0, T]\right), \mu\right)$ be the space of all random variables $F: C_{a, b}[0, T] \rightarrow \mathbb{R}$ such that

$$
\|F\|_{p}=E\left(|F|^{p}\right)^{\frac{1}{p}}<\infty .
$$

Lemma 3.4. For $\phi \in L^{p}(X), p \geq 1$, there exists a sequence $\left\{\xi_{n}\right\}_{n=1}^{\infty}$ in $H(X)$ such that $\phi$ is $\sigma\left(\xi_{n}, n \geq 1\right)$-measurable.

Proof. Let $\left\{e_{n}\right\}_{n=1}^{\infty}$ be a complete orthonormal basis for $L_{a, b}^{1,2}[0, T]$ and let $\xi_{n}=$ $\int_{0}^{T} e_{n}(t) d X_{t}$. Since $1_{[0, t]} \in L_{a, b}^{1,2}[0, T]$ for each $t \in[0, T]$, we have

$$
X_{t}=\int_{0}^{T} 1_{[0, t]}(s) d X_{s}=\sum_{n=1}^{\infty}\left\langle 1_{[0, t]}, e_{n}\right\rangle_{a, b} \int_{0}^{T} e_{n}(s) d X_{s}=\sum_{n=1}^{\infty}\left\langle 1_{[0, t]}, e_{n}\right\rangle_{a, b} \xi_{n} .
$$

Hence $X_{t}$ is $\sigma\left(\xi_{n}, n \geq 1\right)$-measurable for each $t \in[0, T]$. Therefore, $\phi$ is $\sigma\left(\xi_{n}, n \geq 1\right)$ measurable since $\phi$ is $\sigma\left(X_{t}, t \in[0, T]\right)$-measurable.

Theorem 3.5. The set $A=\left\{e^{\xi} \mid \xi \in H(X)\right\}$ is total in $L^{p}(X)$, where $e^{\xi}=$ $L^{p}$-limit of $\sum_{k=0}^{n} \frac{\xi^{k}}{k !}$.

Proof. We first note that each $e^{\xi}$ is an element of $L^{p}(X)$ for each $p \geq 1$. Let $V$ be the $L^{p}$-closure of the linear span of $A$. Suppose $V \neq L^{p}(X)$. Then there exists $\phi \in L^{q}(X)\left(\frac{1}{p}+\frac{1}{q}=1\right)$ such that $\phi \neq 0$ and $E\left[\phi \cdot e^{\xi}\right]=0$ for all $\xi \in H(X)$. Let $\left\{\xi_{n}\right\}$ be as in Lemma 3.4. For any $E \in \sigma\left(\xi_{1}, \xi_{2}, \cdots, \xi_{n}\right) \equiv \mathcal{B}_{n}, \xi_{i} \in H(X)$, $i=1,2, \cdots, n$, we have

$$
\int_{E} \phi \cdot e^{\xi} d \mu=\int_{E} E\left[\phi \cdot e^{\xi} \mid \mathcal{B}_{n}\right] d \mu
$$

for all $\xi \in H(X)$. Hence we have

$$
\int_{C_{a, b}[0, T]} \phi \cdot e^{t_{1} \xi_{1}+\cdots+t_{n} \xi_{n}} d \mu=\int_{C_{a, b}[0, T]} e^{t_{1} \xi_{1}+\cdots+t_{n} \xi_{n}} E\left[\phi \mid \mathcal{B}_{n}\right] d \mu=0 .
$$

By using the Stone-Weierstrass Theorem, one can show that the set $\left\{e^{t_{1} \xi_{1}+\cdots+t_{n} \xi_{n}} \mid\right.$ $\left.t_{i} \in \mathbb{R}, \xi_{i} \in H(X), i=1,2, \cdots, n\right\}$ is total in $L^{p}\left(\xi_{1}, \cdots, \xi_{n}\right)$. This follows because 
$E\left[\phi \mid \mathcal{B}_{n}\right]=0$ a.e. for all $n$. Since $\phi$ is integrable, we have by a well-known martingale convergence theorem and Lemma 3.4 ,

$$
\phi=\lim _{n \rightarrow \infty} E\left[\phi \mid \mathcal{B}_{n}\right]=0 .
$$

This contradiction completes the proof.

Theorem 3.6 (Translation Theorem). Let $F: C_{a, b}[0, T] \rightarrow \mathbb{R}$ be a random variable such that $F \in L^{p}(X)$, and $\gamma(t)=\int_{0}^{t} g(s) d b(s)$ for $g \in L_{b}^{2}[0, T]$. Then

$$
\begin{aligned}
E[F(\cdot+\gamma)] & =E\left[F(\cdot) e^{\int_{0}^{T} g d X(\cdot)-\int_{0}^{T} g d a-\frac{1}{2} \int_{0}^{T} g^{2} d b}\right] \\
& =E\left[F(\cdot) e^{\int_{0}^{T} g d \tilde{X}(\cdot)-\frac{1}{2} \int_{0}^{T} g^{2} d b}\right] .
\end{aligned}
$$

Proof. For $f \in L_{a, b}^{1,2}[0, T]$, let $F \in L^{p}\left(C_{a, b}[0, T]\right)$ be given by $F(x)=e^{\int_{0}^{T} f(t) d X_{t}(x)}$. Then we have

$$
F(x+\gamma)=e^{\int_{0}^{T} f(t) d\left(X_{t}(x)+\gamma(t)\right)}=e^{\int_{0}^{T} f(t) d X_{t}(x)+\int_{0}^{T} f(t) g(t) d b(t)} .
$$

Hence it follows that

$$
E[F(\cdot+\gamma)]=e^{\int_{0}^{T} f d a+\frac{1}{2} \int_{0}^{T} f^{2} d b+\int_{0}^{T} f g d b} .
$$

On the other hand, we have

$$
\begin{aligned}
E\left[F(\cdot) e^{\int_{0}^{T} g d X(\cdot)-\int_{0}^{T} g d a-\frac{1}{2} \int_{0}^{T} g^{2} d b}\right] & =E\left[e^{\int_{0}^{T}(f+g) d X(\cdot)-\int_{0}^{T} g d a-\frac{1}{2} \int_{0}^{T} g^{2} d b}\right] \\
& =e^{\int_{0}^{T} f d a+\frac{1}{2} \int_{0}^{T} f^{2} d b+\int_{0}^{T} f g d b} .
\end{aligned}
$$

Hence the theorem is true for all functionals of the form $e^{\int_{0}^{T} f d X}, f \in L_{a, b}^{1,2}[0, T]$. By Theorem 3.5 the linear span of $\left\{e^{\int_{0}^{T} f d X} \mid f \in L_{a, b}^{1,2}[0, T]\right\}$ is dense in $L^{p}(X)$. Hence the theorem is proved.

\section{INTEGRATION BY PARTS FORMULA FOR $L^{p}$-FUNCTIONALS}

In this section, we define the directional derivative of $L^{p}$-functionals and then use the translation theorem obtained in Section 3 to establish an integration by parts formula for $L^{p}$-functionals.

Definition 4.1. Let $p \geq 1$. The directional derivative of a random variable $F \in$ $L^{p}(X)$ in the direction $\gamma, \gamma(t)=\int_{0}^{t} g(s) d b(s)$ where $g \in L_{b}^{2}[0, T]$, is defined as

$$
\mathcal{D}_{\gamma} F(\cdot)=\lim _{t \rightarrow 0} \frac{1}{t}\{F(\cdot+t \gamma)-F(\cdot)\},
$$

where the limit is taken in the $L^{p}(X)$-sense. Let

$$
\mathcal{D}^{p}(X)=\left\{F \in L^{p}(X) \mid \mathcal{D}_{\gamma} F \text { exists in } L^{p}(X) \text { for all } \gamma \in C_{a, b}^{\prime}[0, T]\right\} .
$$

Lemma 4.2. Let $\epsilon(h)(x)=e^{\int_{0}^{T} h d \tilde{X}(x)-\frac{1}{2} \int_{0}^{T} h^{2} d b}, h \in L_{b}^{2}[0, T]$, be a functional on $C_{b}[0, T]$. Then $\epsilon(h) \in L^{p}(X)$ for all $p \geq 1$. Moreover, for any $p \geq 1$,

$$
\lim _{t \rightarrow 0} \frac{1}{t}\{\epsilon(t h)-1\}=\int_{0}^{T} h d \tilde{X}
$$

where the limit is taken in the $L^{p}(X)$-sense. 
Proof. Let $h \in L_{b}^{2}[0, T]$. We first note that $e^{\left|\int_{0}^{T} h d \tilde{X}\right|} \in L^{p}(X)$ for all $p \geq 1$. So we see that $\|\epsilon(h)\|_{p}<\infty$. Let $A>0$ be fixed. Define a function $f$ on $C_{a, b}[0, T] \times[-A, A]$ by

$$
f(x, t)=\epsilon(t h)(x)=e^{t \int_{0}^{T} h d \tilde{X}(x)-\frac{t^{2}}{2} \int_{0}^{T} h^{2} d b} .
$$

Then for almost all $x \in C_{a, b}[0, T], f(x, \cdot)$ is differentiable on $(-A, A)$ and

$$
\frac{\partial f}{\partial t}(x, t)=\left(\int_{0}^{T} h d \tilde{X}-t \int_{0}^{T} h^{2} d b\right) f(x, t) .
$$

If $-A<t<A$, then by the mean value theorem, for each $x \in C_{a, b}[0, T]$ there exists $\theta \equiv \theta(x), 0<\theta<1$, such that

$$
\frac{1}{t}(f(x, t)-f(x, 0))=\frac{\partial f}{\partial t}(x, \theta t) .
$$

From this fact and the inequalities $\left|\int_{0}^{T} h d \tilde{X}\right| \leq e^{\left|\int_{0}^{T} h d \tilde{X}\right|}$ and $\left|e^{u}\right| \leq e^{|u|}$ for $u \in \mathbb{R}$, we have the following inequalities:

$$
\begin{aligned}
& \left|\frac{1}{t}\{\epsilon(t h)(x)-1\}-\int_{0}^{T} h d \tilde{X}(x)\right| \\
& \quad=\left|\left\{\left(\int_{0}^{T} h d \tilde{X}(x)-\theta t \int_{0}^{T} h^{2} d b\right) \cdot \epsilon(\theta t h)(x)\right\}-\int_{0}^{T} h d \tilde{X}(x)\right| \\
& \leq \alpha e^{\beta\left|\int_{0}^{T} h d \tilde{X}(x)\right|}
\end{aligned}
$$

for some constants $\alpha>0$ and $\beta>0$. Since $\alpha e^{\beta\left|\int_{0}^{T} h d \tilde{X}\right|} \in L^{p}(X)$ for all $p \geq 1$, by the dominated convergence theorem,

$$
\lim _{t \rightarrow 0} E\left[\left|\frac{1}{t}(\epsilon(t h)-1)-\int_{0}^{T} h d \tilde{X}\right|^{p}\right]=0
$$

and the lemma is proved.

Theorem 4.3. Let $F \in \mathcal{D}^{p}(X)$ and $\gamma(t)=\int_{0}^{t} g(u) d b(u)$ with $g \in L_{a, b}^{1,2}[0, T]$. Then

$$
E\left[\mathcal{D}_{\gamma} F\right]=E\left[F \cdot \int_{0}^{T} g d X\right]-\int_{0}^{T} g d a \cdot E[F] .
$$

Proof. Since $F \in \mathcal{D}^{p}, \frac{1}{t}\{F(\cdot+t \gamma)-F(\cdot)\}$ converges to $\mathcal{D}_{\gamma} F$ in $L^{p}(X)$. Hence it converges to $\mathcal{D}_{\gamma} F$ in $L^{1}(X)$. From Theorem 3.6 and Lemma 4.2, it follows that

$$
\begin{aligned}
E\left[\mathcal{D}_{\gamma} F\right] & =\lim _{t \rightarrow 0} E\left[\frac{1}{t}\{F(\cdot+t \gamma)-F(\cdot)\}\right] \\
& =\lim _{t \rightarrow 0} E\left[F(\cdot)\left\{\frac{1}{t}(\epsilon(t g)-1)\right\}\right] \\
& =E\left[F \cdot\left(\int_{0}^{T} g d X-\int_{0}^{T} g d a\right)\right]
\end{aligned}
$$

and the theorem is proved.

Theorem 4.4 (Integration by parts formula). Let $F \in \mathcal{D}^{p}(X), G \in \mathcal{D}^{q}(X)$ with $\frac{1}{p}+\frac{1}{q}=1$. Then we have, for $\gamma(t)=\int_{0}^{t} g(u) d b(u)$ with $g \in L_{a, b}^{1,2}[0, T]$,

$$
E\left[G \cdot \mathcal{D}_{\gamma} F\right]=E\left[F \cdot G \cdot \int_{0}^{T} g d X\right]-\int_{0}^{T} g d a \cdot E[F \cdot G]-E\left[F \cdot \mathcal{D}_{\gamma} G\right] .
$$


Proof. We first note that for $F \in L^{p}(X)$ and $G \in L^{q}(X)$ with $\frac{1}{p}+\frac{1}{q}=1$, we have $F \cdot G \in L^{1}(X)$. Now we shall show that the limit

$$
\lim _{t \rightarrow 0} \frac{1}{t}\{F(\cdot+t \gamma) \cdot G(\cdot+t \gamma)-F(\cdot) \cdot G(\cdot)\}
$$

exists in $L^{1}(X)$ and the limit is equal to $G \cdot \mathcal{D}_{\gamma} F+F \cdot \mathcal{D}_{\gamma} G$. This assertion follows from the following inequality:

$$
\begin{aligned}
& E\left[\left|\frac{1}{t}\{F(\cdot+t \gamma) \cdot G(\cdot+t \gamma)-F(\cdot) \cdot G(\cdot)\}-\left\{G \cdot \mathcal{D}_{\gamma} F+F \cdot \mathcal{D}_{\gamma} G\right\}\right|\right] \\
& \leq\left\|\left\{\frac{1}{t}(F(\cdot+t \gamma)-F(\cdot))-\mathcal{D}_{\gamma} F\right\} G(\cdot+t \gamma)\right\|_{1}+\left\|\mathcal{D}_{\gamma} F\{G(\cdot+t \gamma)-G(\cdot)\}\right\|_{1} \\
& +\left\|F\left\{\frac{1}{t}(G(\cdot+t \gamma)-G(\cdot))-\mathcal{D}_{\gamma} G\right\}\right\|_{1} \\
& \leq\left\|\frac{1}{t}(F(\cdot+t \gamma)-F(\cdot))-\mathcal{D}_{\gamma} F\right\|_{p}\|G(\cdot+t \gamma)\|_{q}+\left\|\mathcal{D}_{\gamma} F\right\|_{p}\|G(\cdot+t \gamma)-G(\cdot)\|_{q} \\
& +\|F\|_{p}\left\|\frac{1}{t}(G(\cdot+t \gamma)-G(\cdot))-\mathcal{D}_{\gamma} G\right\|_{q}
\end{aligned}
$$

since all three terms on the right-hand side above go to zero as $t$ goes to zero. It then follows that

$$
E\left[\mathcal{D}_{\gamma}(F \cdot G)\right]=E\left[G \cdot \mathcal{D}_{\gamma} F\right]+E\left[F \cdot \mathcal{D}_{\gamma} G\right]
$$

But this implies that $F \cdot G \in \mathcal{D}^{1}(X)$, and hence by Theorem 4.3 ,

$$
E\left[\mathcal{D}_{\gamma}(F \cdot G)\right]=E\left[(F \cdot G) \cdot \int_{0}^{T} g d X\right]-\int_{0}^{T} g d a E[F \cdot G] .
$$

Therefore, we conclude that

$$
E\left[G \cdot \mathcal{D}_{\gamma} F\right]=E\left[(F \cdot G) \cdot \int_{0}^{T} g d X\right]-\int_{0}^{T} g d a E[F \cdot G]-E\left[F \cdot \mathcal{D}_{\gamma} G\right]
$$

and the theorem is proved.

\section{ACKNOWLEDGEMENT}

The author thanks Professor Dong Myung Chung for his valuable discussion on this problem.

\section{REFERENCES}

1. N. Aronszajn, Theory of reproducing kernels, Trans. Amer. Math. Soc. 68(1950), 337-404. MR0051437(14:479c)

2. R. H. Cameron and D. A. Storvick, Feynman integral of variations of functions, Gaussian random fields, Ser. Prob. Statist. 1(1991), 144-157. MR1163606 (93b:28035)

3. S. J. Chang and D. M. Chung, Conditional function space integrals with applications, Rocky Mountain J. of Math. 26(1)(1996), 37-62. MF1386151 (97d:28014)

4. S. J. Chang, J. G. Choi and D. Skoug, Integration by parts formulas involving generalized Fourier-Feynman transforms on function space, Trans. Amer. Math. Soc.,355(2003), 29252948. MR1975406 (2004c:60229)

5. S. J. Chang and D. Skoug, Generalized Fourier-Feynman transforms and a first variation on function space, Integral Transforms and Special Functions, Vol 14, No 5(2003), 375-393. MR2005996 (2004h:81098)

6. G. Kallianpur, The role of reproducing kernel Hilbert spaces in the study of Gaussian processes, Advances in Probability and Related Topics 2(P. Ney,ed.), (1970), Marcel Dekker, New York. MR0283866 (44:1096) 
7. E. Kreyszig, Introductory functional analysis with applications, John Wiley and Sons. Inc., 1978. MR0467220 (57:7084)

8. H.-H. Kuo, Gaussian measures in Banach spaces, Lect. Notes in Math. Vol.463, SpringerVerlag, 1975. MR 0461643 (57:1628)

9. D. Lamberton and B. Lapeyre, Introduction to stochastic calculus applied to finance, Chapman and Hall, 1996. MR1422250 (98b:90018)

10. B. Oksendal, An introduction to Malliavin calculus with applications to economics, Working paper, No 3/96, Norwegian School of Economics and Business Administration, 1996.

11. B. Oksendal, Stochastic differential equations, Springer-Verlag (fifth edition), 2000.

12. J. Yeh, Stochastic process and the Wiener integral, Marcel Dekker, Inc., New York, 1973. MR0474528 (57:14166)

Department of Mathematics, Sogang University, Seoul 121-742, Korea

E-mail address: rouge@sogang.ac.kr

Current address: Courant Institute of Mathematical Sciences, 251 Mercer Street, New York, New York 10012 